\title{
IDENTIFICATION OF STEADY AND NON-STEADY GAIT OF HUMAN- EXOSKELETON WALKING SYSTEM
}

\author{
K.K. ŻUR \\ Faculty of Management \\ Bialystok University of Technology \\ Ojca Tarasiuka street 2, 16-001 Kleosin, POLAND \\ Faculty of Mechanical Engineering \\ Bialystok University of Technology \\ Wiejska street 45C, 15-351 Bialystok, POLAND \\ E-mail:k.zur@pb.edu.pl
}

\begin{abstract}
In this paper a method of analysis of exoskeleton multistep locomotion was presented by using a computer with the preinstalled DChC program. The paper also presents a way to analytically calculate the ",motion indicator", as well as the algorithm calculating its two derivatives. The algorithm developed by the author processes data collected from the investigation and then a program presents the obtained final results. Research into steady and non-steady multistep locomotion can be used to design two-legged robots of DAR type and exoskeleton control system.
\end{abstract}

Key words: identification, steady gait, non-steady gait, exoskeleton.

\section{Introduction}

In the $20^{\text {th }}$ century most scientists concentrated on research into human gait carried out on a flat surface, usually in laboratory conditions. This research focused on relatively constant-frequency and constant-velocity aspect of human gait. It appears that contemporary optoelectronic systems of gait analysis enable estimation of at most two steps (usually one) of a moving person (subject), and so far, graphs of the necessary amount of power developed in multistep human gait have not been analyzed (Jaworek, 1992).

At present only electrogoniometers allow examination of multistep locomotion in both: laboratory and field conditions (Jaworek and Ferenc, 1992; Jaworek et al., 1998). Previous research into the use of electrogoniometers was also focused on relatively constant velocity and constant frequency of human gait.

Multistep locomotion with changing (in time) velocity and frequency is still a problem in biomechanics of human locomotion. It can be solved by using the motion indicator $\varepsilon_{a_{p-/+}}$ applied in another paper (Jaworek, 2006) and its first and second time derivatives. Changes of the value in time of those quantities allow classification of human locomotion into one of the groups (steady or non-steady locomotion) in relation to the demand of the amount of average power used during multistep gait with variable frequency and velocity.

This type of research is significant in rehabilitation of lower limbs because it is claimed that not every subject is capable of one type of locomotion.

Moreover, research into non-constant-velocity and non-constant-frequency multistep locomotion can contribute to broadening the knowledge about the gait mechanism of two-legged creatures and estimated research results can be used to design two-legged anthropomorphic robots (and four-legged galloping machines) control systems. 


\section{Types of human gait}

Table 1 below introduces steady, non-steady and mixed gait of a human being in relation to value change of the first and second time derivative of motion indicator $\varepsilon_{a_{p-/+}}$ (Jaworek, 2006).

Table 1. Classification of gait.

\begin{tabular}{|c|c|c|c|}
\hline \multicolumn{2}{|c|}{ TYPES OF HUMAN GAIT } & $\dot{\bullet}_{a_{p-/+}}$ & $\ddot{\varepsilon}_{a_{p-/+}}$ \\
\hline \multirow{3}{*}{ STEADY LOCOMOTION } & $\begin{array}{l}\text { with constant demand } \\
\text { for medium power }\end{array}$ & $=0$ & $=0$ \\
\hline & $\begin{array}{c}\text { with linear increase of } \\
\text { demand for medium power }\end{array}$ & $=$ const $>0$ & $=0$ \\
\hline & $\begin{array}{c}\text { with linear decrease of } \\
\text { demand for medium power }\end{array}$ & $=$ const $<0$ & $=0$ \\
\hline \multirow{2}{*}{ NON-STEADY LOCOMOTION } & $\begin{array}{l}\text { chaotically determined locomotion } \\
\text { (with nonlinear demand for medium power) }\end{array}$ & $\neq$ const & $\begin{aligned}= & \text { const } \neq 0 \\
& \neq \text { const }\end{aligned}$ \\
\hline & $\begin{array}{l}\text { chaotically undetermined locomotion } \\
\text { (with nonlinear demand for medium power) }\end{array}$ & $\neq$ const & $\begin{aligned}= & \text { const } \neq 0 \\
& \neq \text { const }\end{aligned}$ \\
\hline \multicolumn{2}{|c|}{ MIXED GAIT } & \multicolumn{2}{|c|}{$\begin{array}{c}=0 \\
=\text { const } \neq 0 \\
\neq \text { const }\end{array}$} \\
\hline
\end{tabular}

In the case of steady locomotion the demand for average power developed by main muscular systems supporting main joints of a human leg during gait might linearily increase, linearily decrease or it is constant. In the case of non-steady locomotion the demand for average power is nonlinear. Non-steady chaotic locomotion is divided into two parts: determined and undetermined in relation to changes of the first derivative of the motion indicator $\varepsilon_{a_{p-/+}}$.

It should also be noticed that in steady locomotion the second derivative of motion indicator is always equal to zero while the second derivative in non-steady locomotion is always a number other than zero.

\section{Analytic determination of time derivatives of motion indicator}

The motion indicator (a new notion, earlier presented as the index of movement) $\varepsilon_{a_{p-/+}}$ is defined as follows

$$
\varepsilon_{a_{p-/+}}=\frac{P_{-/+}}{a_{p} \cdot m \cdot v}, \quad \text { no unit }
$$

where:

$P_{-/+}-$medium power developed by hip, knee and ankle joints of a man during support and swing phase, $W$; $m$ - body mass of a man, $\mathrm{kg}$; 
$\bar{v}$ - medium velocity in sagittal plane of gait, $m / s$;

$a_{p}$ - extensity of gravitational field of the Earth, $m / s^{2}$.

The motion indicator $\varepsilon_{a_{p-/+}}$ can be interpreted physically as follows: it is the amount of average power, which is necessary to carry $1 \mathrm{~kg}$ of biomass on limbs on the planet Earth, with extensity of gravitational field which equals $a_{p}$, with medium velocity equal to $1 \mathrm{~m} / \mathrm{s}$.

An approximated formula for the amount of medium power $P_{-I_{+}}$in complete gait cycle is defined as follows

$$
P_{-/+}=2.1 \cdot m \cdot v^{\alpha} \cdot f^{\beta}+3.4 \cdot \zeta^{\gamma}-1.1, \quad[W]
$$

where:

$P_{-/+}-$medium power developed by three main muscle systems operating the hip, ankle and knee joints of a human leg during the support phase of gait and by two main muscle systems operating the hip and knee joints of human leg during the swing phase of gait, $W$;

$m$ - body mass of a man, $\mathrm{kg}$;

$\bar{v}$ - medium velocity in sagittal plane of gait, $\mathrm{m} / \mathrm{s}$;

$f$ - frequency of gait (quotient of $n_{p}$ and time $t$ ), where: $n_{p}$ - relatively constant number of single steps taken by an investigated subject on the measurement footpath of length $s \in[11 \div 13 \mathrm{~m}]$ in period of time $t$;

$\zeta$-normalized (non-dimensional) surface defined by the difference in surface areas of small and huge loop on phase diagram appropriate for the hip and ankle joints of a human leg; for the full gait cycle $\zeta \in[1 \div 10]$, for values of velocity and frequency in the range of their change from minimal to maximal values during gait in sagittal plane;

$\alpha, \beta, \gamma$-constant values (non-dimensional); in the case of gait of a healthy subject on a relatively flat and hardened surface should be $\alpha=\beta=\gamma=1$;

2.1 - constant factor with no physical interpretation, $\mathrm{m} / \mathrm{s}$;

3.4,1.1 - constant factors with no physical interpretation, $W$.

The first element of sum formula (3.2) is a physical model of medium power developed by the muscle system of a lower limb of healthy, young or mature subjects during the support phase of their gait. The second element is a model of medium power developed by muscle systems of the limbs of the same subjects during the swing phase of their gait.

The time relation of two derivatives is described by the equations below:

a) first derivative of motion with respect to time indicator is

$$
\dot{\varepsilon}_{a_{p-1+}}=F_{1}^{*}(\cdot)-\frac{d \bar{v}}{d t} \cdot F_{2}^{* *}(\cdot), \quad[1 / s]
$$

where

$$
F_{1}^{*}(\cdot)=\frac{1}{a_{p}}\left[2.1 \cdot \frac{d f}{d t}+3.4 \cdot \frac{\frac{d \zeta}{d t}}{p}\right], \quad[1 / s]
$$




$$
F_{2}^{* *}(\cdot)=\frac{1}{a_{p}}\left[1.7 \cdot \frac{\zeta}{\overline{E_{k}}}-0.55 \cdot \frac{1}{\overline{E_{k}}}\right], \quad[\mathrm{s} / \mathrm{m}]
$$

b) second derivative of motion indicator is

$$
\ddot{\varepsilon}_{a_{p-++}}=\dot{F}_{1}^{*}(\cdot)-\frac{d^{2} \bar{v}}{d t^{2}} \cdot F_{2}^{* *}(\cdot)-\frac{d \bar{v}}{d t} \cdot \dot{F}_{2}^{* *}(\cdot), \quad\left[1 / s^{2}\right]
$$

where

$$
\begin{array}{ll}
\dot{F}_{l}^{*}(\cdot)=\frac{1}{a_{p}} \cdot\left[2.1 \cdot \frac{d^{2} f}{d t^{2}}+3.4 \cdot \frac{\frac{d^{2} \zeta}{d t^{2}} \cdot \bar{p}-\frac{d \zeta}{d t} \cdot \frac{d \bar{p}}{d t}}{(\bar{p})^{2}}\right], & {\left[1 / s^{2}\right],} \\
\ddot{F}_{2}^{* *}(\cdot)=\frac{1}{a_{p}}\left[1.7 \cdot \frac{\frac{d \zeta}{d t} \cdot \overline{E_{k}}-\zeta \cdot \frac{d \overline{E_{k}}}{d t}}{\left(\overline{E_{k}}\right)^{2}}+0.55 \cdot \frac{\frac{d \overline{E_{k}}}{d t}}{\left(\overline{E_{k}}\right)^{2}}\right], & {[1 / m] .}
\end{array}
$$

Individual notations used in Eqs $(3 \div 3.8)$ are defined as follows (in order of appearance): $a_{p}=$ const - gravitational acceleration of the planet Earth, $\mathrm{m} / \mathrm{s}^{2}$;

$\frac{d \bar{v}}{d t}=\lim _{\Delta t \rightarrow 0}\left(\frac{\Delta \bar{v}}{\Delta t}\right)=\frac{\bar{v}-\overline{v_{0}}}{T_{-/+}}-$acceleration of the subject in sagittal plane of movement, $\mathrm{m} / \mathrm{s}^{2} ;$

$\bar{v}$ - average velocity of the subject in sagittal plane, at present, $\mathrm{m} / \mathrm{s}$;

$\overline{v_{0}}$-average velocity of the subject, in the past, $\mathrm{m} / \mathrm{s}$;

$T_{-/+}$- time of stride, $s$;

$\frac{d f}{d t}=\lim _{\Delta t \rightarrow 0}\left(\frac{\Delta f}{\Delta t}\right)=\frac{f-f_{o}}{T_{-/+}}-$first derivative of subject's gait frequency, $1 / s^{2}$;

$f$ - gait frequency of the subject, in present time, $1 / s$;

$f_{0}$ - gait frequency of the subject, in past time, $1 / \mathrm{s}$;

$\frac{d \zeta}{d t}=\frac{d \zeta}{d f} \cdot \frac{d f}{d t}-$ first derivative of surface $\zeta, 1 / s ;$

$\frac{d \zeta}{d f}=\lim _{\Delta f \rightarrow 0}\left(\frac{\Delta \zeta}{\Delta f}\right)=\frac{\zeta-\zeta_{0}}{f-f_{0}}-$ first frequency derivative of surface $\zeta, s ;$

$\zeta$ surface derived from the difference between big and small surface in phase diagram from the hip and knee joints of a human leg during gait, in present time, no unit;

$\zeta_{0}$ - surface $\zeta$, in past time, no unit;

$\bar{p}=m \cdot \bar{v}$-average momentum of the subject, $\mathrm{kg} \cdot \mathrm{m} / \mathrm{s}$;

$\mathrm{m}$ - body mass of a man, $\mathrm{kg}$; 
$\overline{E_{k}}=1 / 2 \cdot m \cdot(\bar{v})^{2}-$ average kinetic energy of a moving subject, $J$;

$\frac{d^{2} \bar{v}}{d t^{2}}=\lim _{\Delta t \rightarrow 0}\left(\frac{\Delta \frac{d \bar{v}}{d t}}{\Delta t}\right)=\frac{\frac{d \bar{v}}{d t}-\frac{d \bar{v}}{d t} 0}{T_{-/+}}-$first derivative of acceleration $\frac{d \bar{v}}{d t}, \mathrm{~m} / \mathrm{s}^{3} ;$

$\frac{d \bar{v}}{d t_{0}}-$ acceleration, in past time, $\mathrm{m} / \mathrm{s}^{2}$;

$\frac{d^{2} f}{d t^{2}}=\lim _{\Delta t \rightarrow 0}\left(\frac{\Delta \frac{d f}{d t}}{\Delta t}\right)=\frac{\frac{d f}{d t}-\frac{d f}{d t} 0}{T_{-/+}}-$second derivative of frequency, $1 / \mathrm{s}^{3} ;$

$\frac{d f}{d t_{0}}$ - first derivative of frequency, in past time, $1 / s^{2}$;

$\frac{d^{2} \zeta}{d t^{2}}=\frac{d^{2} \zeta}{d t d f} \cdot \frac{d f}{d t}+\frac{d \zeta}{d t} \cdot \frac{d^{2} f}{d t^{2}}-$ second derivative of surface $\zeta, 1 / s^{2}$

$\frac{d^{2} \zeta}{d t d f}=\lim _{\Delta t \rightarrow 0}\left(\frac{\Delta \frac{d \zeta}{d f}}{\Delta t}\right)=\frac{\frac{d \zeta}{d f}-\frac{d \zeta}{d f_{0}}}{T_{-/+}}-$mixed second derivative of surface $\zeta$, no unit;

$\frac{d \zeta}{d f_{0}}-$ first frequency derivative of surface $\zeta$, in past time, $s$;

$\frac{d \bar{p}}{d t}=\lim _{\Delta t \rightarrow 0}\left(\frac{\Delta \bar{p}}{\Delta t}\right)=\frac{\bar{p}-\bar{p}_{0}}{T_{-/+}}-$first derivative of average momentum $\bar{p}, \mathrm{~kg} \cdot \mathrm{m} / \mathrm{s}^{2}$;

$\bar{p}_{0}$-average momentum $\bar{p}$, in past time, $\mathrm{kg} \cdot \mathrm{m} / \mathrm{s}$;

$\frac{d \overline{E_{k}}}{d t}=\lim _{\Delta t \rightarrow 0}\left(\frac{\Delta \overline{E_{k}}}{\Delta t}\right)=\frac{\overline{E_{k}}-\overline{E_{k}} 0}{T_{-/+}}-$first derivative of average kinetic energy $\overline{E_{k}}, \mathrm{~J} / \mathrm{s}$;

$\overline{E_{k 0}}$ - average kinetic energy, in past time, $J$;

2.1 - constant coefficient with no physical interpretation, $\mathrm{m} / \mathrm{s}$;

$3.4,1.7,0.55$ - constant coefficients with no physical interpretation, $W$.

Moreover, to determine the surface $\zeta$ which appears in Eq.(3.8) we must use the relation (3.2) and transform it into

$$
\zeta=\frac{P_{-1+}-2.1 \cdot m \cdot \bar{v} \cdot f+1.1}{3.4}
$$

Time derivatives of the motion indicator are graphs in discrete time $t$, which is derived from the following equation

$$
t_{n}=\sum_{t=0}^{n} T_{i-/+}
$$




\section{DChC programme}

In order to facilitate calculations of two motion indicators time derivatives and also for visualization, the authors' own program DChC was developed. With its aid we can define graphs of derivatives of any human gait if we have at least 3 to 5 analyzed steps (with variable velocity and frequency) of the same person. With data from separate analyses we can determine various variants of gait of the subject (the program memorizes data with the help of line tables); for example, from maximum to minimum velocity, from minimum across maximum to minimum velocity again. We can also determine the exact velocity constant and constant frequency of movement. The final result of the program operation is generation of time graphs of the motion indicator and its two time derivatives.

The DChC program is a modification of the software called the AChC and it was written in Delphi programming language. The main algorithm of the program is shown in Fig1.

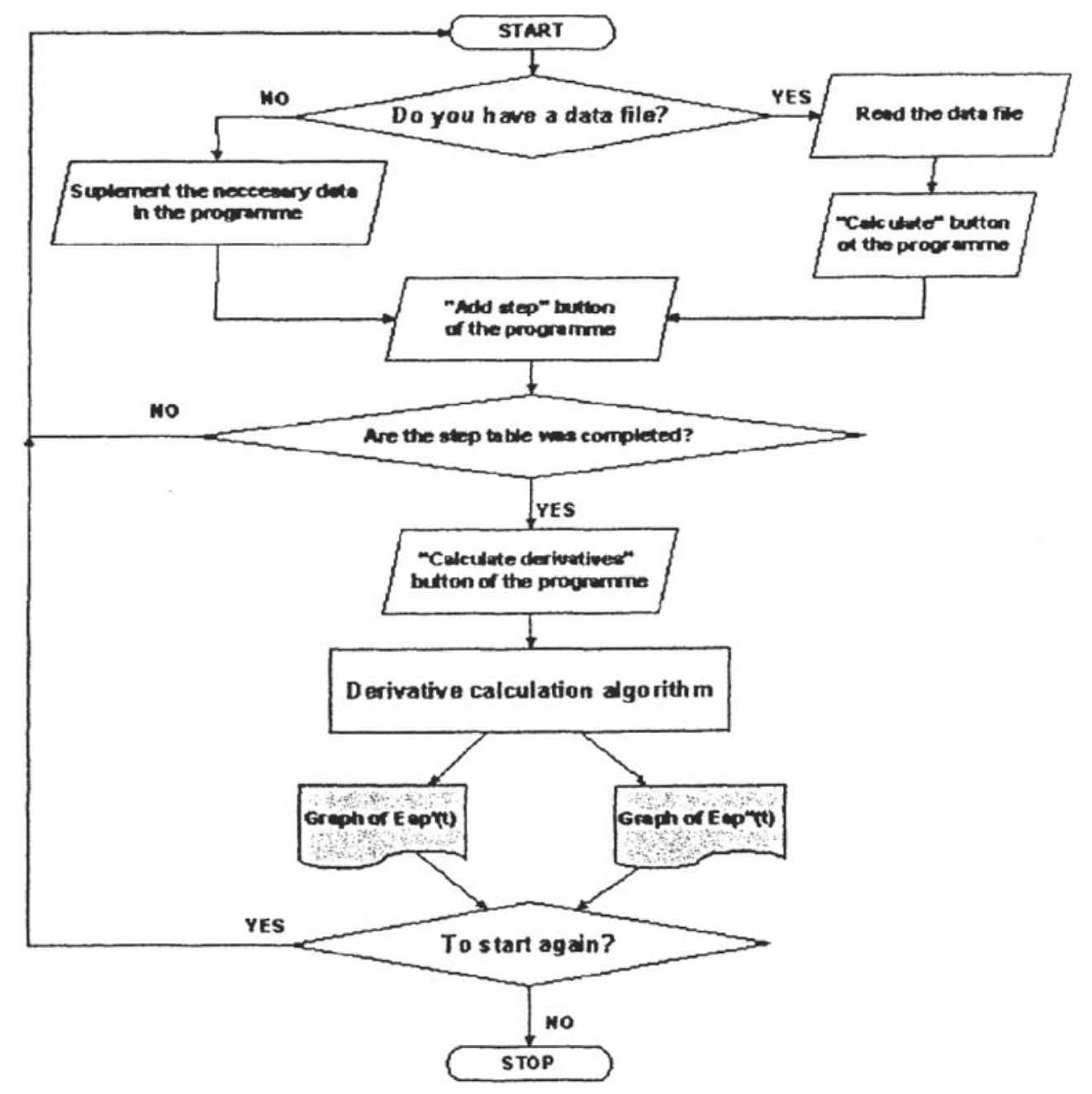

Fig.1. Main algorithm of the DChC programme. 
A detailed algorithm of determining two derivatives of the motion indicator is shown below in Fig. 2 .

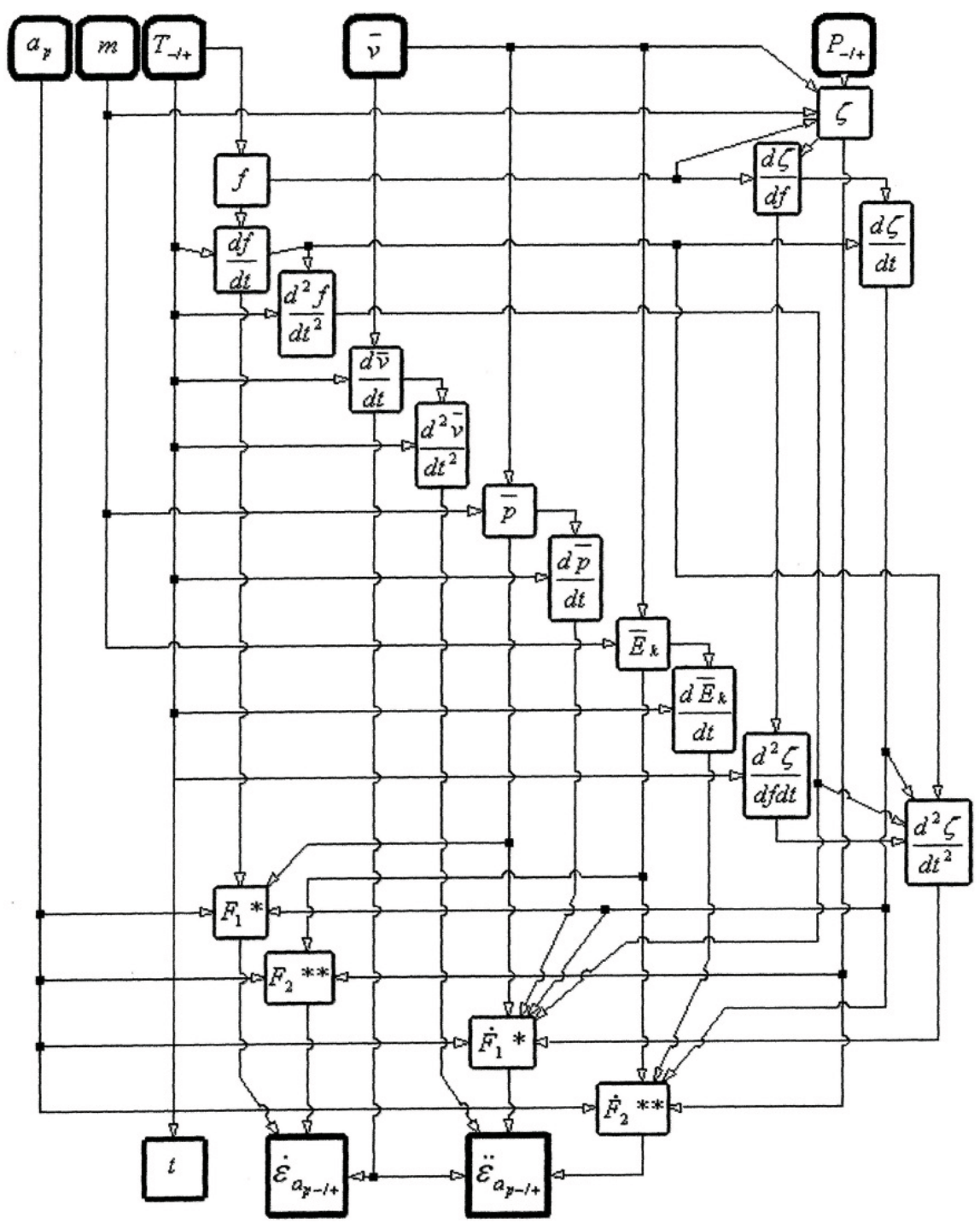

Fig.2. Detailed algorithm of the DChC program.

All values from the diagram mentioned above excluding $a_{p}$ and $m$ are multi line matrixes. A way of determining sequential values was programmed according to Eqs (3.3) and (3.6) and its developed elements. Calculations were carried out with aid of loops defined in the program's code. The values of the first (3.3) and second (3.6) time derivative of the motion indicator are shown in dependence of time $t$, which is derived from Eq.(3.10).

\subsection{Examples of the DChC program output}

The data $(w 1, w 2, w 3)$ are from David Winter's book and show generalized slow, natural and fast motion of 17-19 subjects with average body mass of 70 kilograms (Tab.2).

Other data are from the research carried out in Bioengineering Center in Milan and concerning one subject - a woman with the body mass of $58 \mathrm{~kg}$ and body height of $160 \mathrm{~cm}$ (Winter, 1991). 
Table 2. Data used in examples (Winter, 1991).

\begin{tabular}{|c|c|c|c|c|c|}
\hline & & $\begin{array}{c}\bar{v} \\
m / s\end{array}$ & $\begin{array}{c}T_{-/+} \\
S\end{array}$ & $\begin{array}{c}P_{-/+} \\
W\end{array}$ & $\begin{array}{l}\varepsilon_{a_{p-/+}} \\
\text { no unit }\end{array}$ \\
\hline & $w 1$ & $\begin{array}{r}0.998 \\
\pm 0.006\end{array}$ & $\begin{array}{l}1.383 \\
\pm 0.002\end{array}$ & $\begin{array}{l}65.342 \\
\pm 2.182\end{array}$ & $\begin{array}{c}0.095 \\
\pm 0.004\end{array}$ \\
\hline $\begin{array}{c}\text { data } \\
m=70 \mathrm{~kg}\end{array}$ & $w 2$ & $\begin{array}{l}1.325 \\
\pm 0.006\end{array}$ & $\begin{array}{c}1.140 \\
\pm 0.002\end{array}$ & $\begin{array}{c}108.679 \\
\pm 2.235\end{array}$ & $\begin{array}{c}0.119 \\
\pm 0.003\end{array}$ \\
\hline & $w 3$ & $\begin{array}{l}1.682 \\
\pm 0.006\end{array}$ & $\begin{array}{l}0.975 \\
\pm 0.002\end{array}$ & $\begin{array}{l}180.109 \\
\pm 2.329\end{array}$ & $\begin{array}{c}0.156 \\
\pm 0.003\end{array}$ \\
\hline & $k 1$ & $\begin{array}{l}1.003 \\
\pm 0.006\end{array}$ & $\begin{array}{l}1.280 \\
\pm 0.002\end{array}$ & $\begin{array}{l}66.960 \\
\pm 1.712\end{array}$ & $\begin{array}{c}0.117 \\
\pm 0.004\end{array}$ \\
\hline Woman & $k 2$ & $\begin{array}{l}1.246 \\
\pm 0.006\end{array}$ & $\begin{array}{l}1.120 \\
\pm 0.002\end{array}$ & $\begin{array}{c}103.200 \\
\pm 1.777\end{array}$ & $\begin{array}{c}0.146 \\
\pm 0.004\end{array}$ \\
\hline$M=58 \mathrm{~kg}$ & $k 3$ & $\begin{array}{l}1.328 \\
\pm 0.006\end{array}$ & $\begin{array}{l}1.080 \\
\pm 0.002\end{array}$ & $\begin{array}{l}110.450 \\
\pm 1.803\end{array}$ & $\begin{array}{c}0.146 \\
\pm 0.004\end{array}$ \\
\hline & $k 4$ & $\begin{array}{c}1.602 \\
\pm 0.006\end{array}$ & $\begin{array}{c}0.980 \\
\pm 0.002\end{array}$ & $\begin{array}{c}155.410 \\
\pm 1.896\end{array}$ & $\begin{array}{c}0.170 \\
\pm 0.003\end{array}$ \\
\hline
\end{tabular}

The program for 'designing human gait' was developed according to the rules shown in Tab.3.

Table 3. "Designing" of multistep two-legged human locomotion by using proper steps from Tab.2.

\begin{tabular}{|c|c|c|c|c|c|c|}
\hline \multicolumn{2}{|c|}{} & \multicolumn{5}{|c|}{ STEP } \\
\cline { 2 - 7 } \multicolumn{2}{|c|}{} & $\mathbf{1}$ & $\mathbf{2}$ & $\mathbf{3}$ & $\mathbf{4}$ & $\mathbf{5}$ \\
\hline \multirow{4}{*}{ Example } & $\mathbf{1}$ & $k 4$ & $k 4$ & $k 3$ & $k 2$ & $k 1$ \\
\cline { 2 - 7 } & $\mathbf{2}$ & $w 3$ & $w 3$ & $w 3$ & $w 1$ & $w 3$ \\
\cline { 2 - 7 } & $\mathbf{3}$ & $w 2$ & $w 2$ & $w 2$ & $w 2$ & $w 2$ \\
\cline { 2 - 7 } & $\mathbf{4}$ & $w 1$ & $w 1$ & $w 1$ & $w 2$ & $w 3$ \\
\hline
\end{tabular}

Table 3 ought to be read as follows: when the program asks us to write data for step 3 and we are operating on example 4 then we fill the appropriate spaces (in the program's interface) with data from Tab. 2 marked as $w 1$.

The results obtained from the DChC program are shown in Figs 3 to 6. 

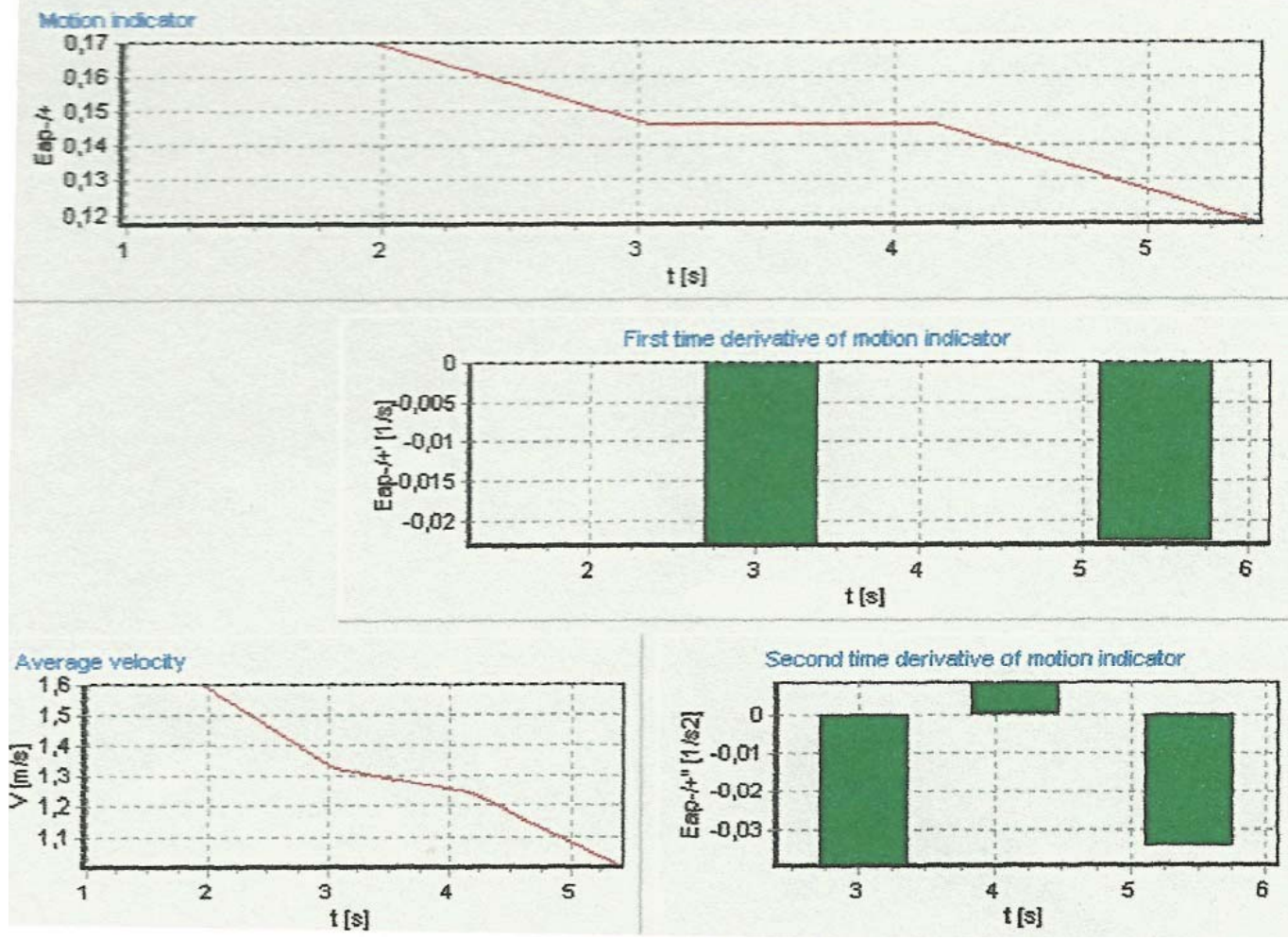

Fig.3. Example 1 - non-steady locomotion, chaotically determined.
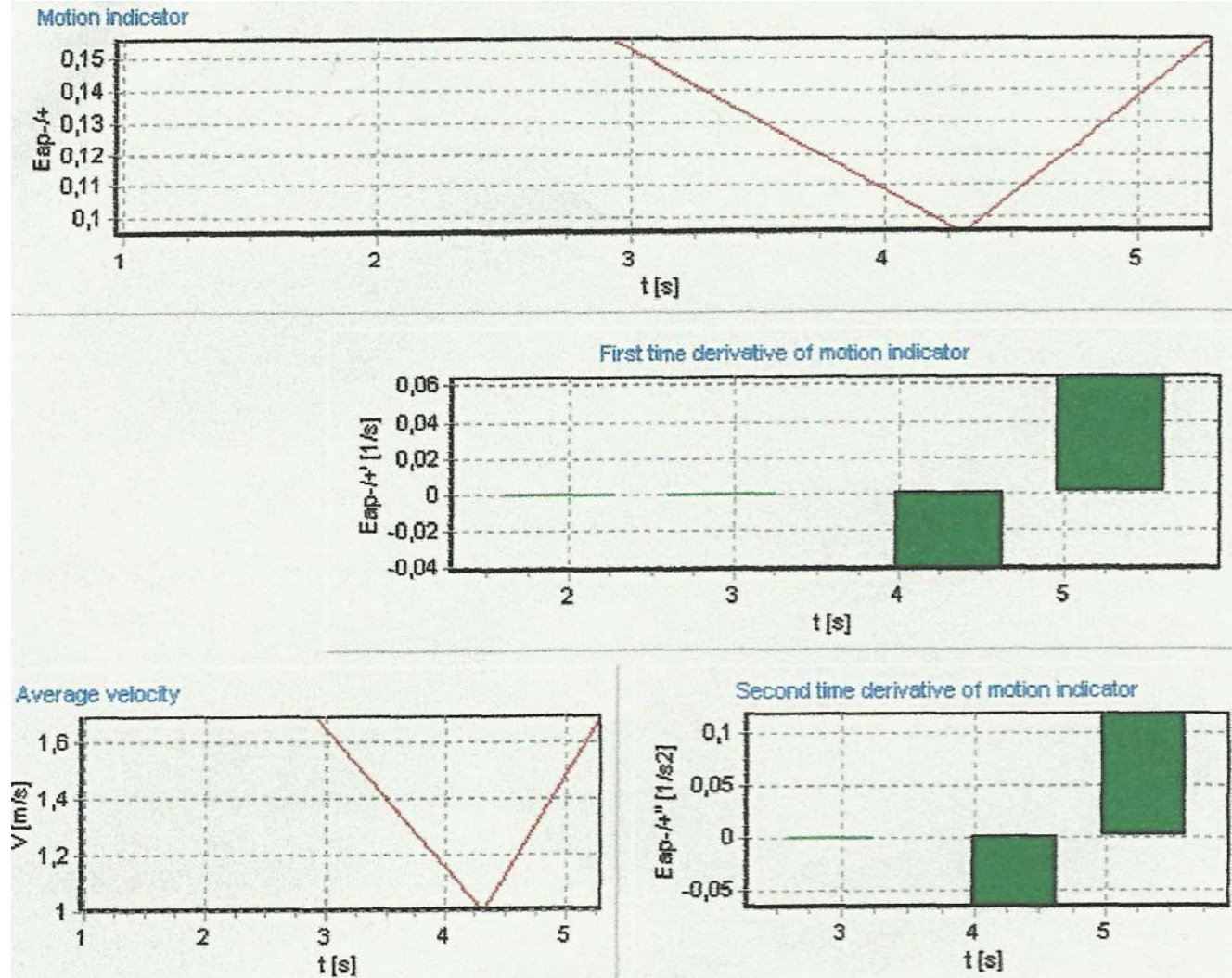

Fig.4. Example 2 - non-steady locomotion, chaotically determined. 

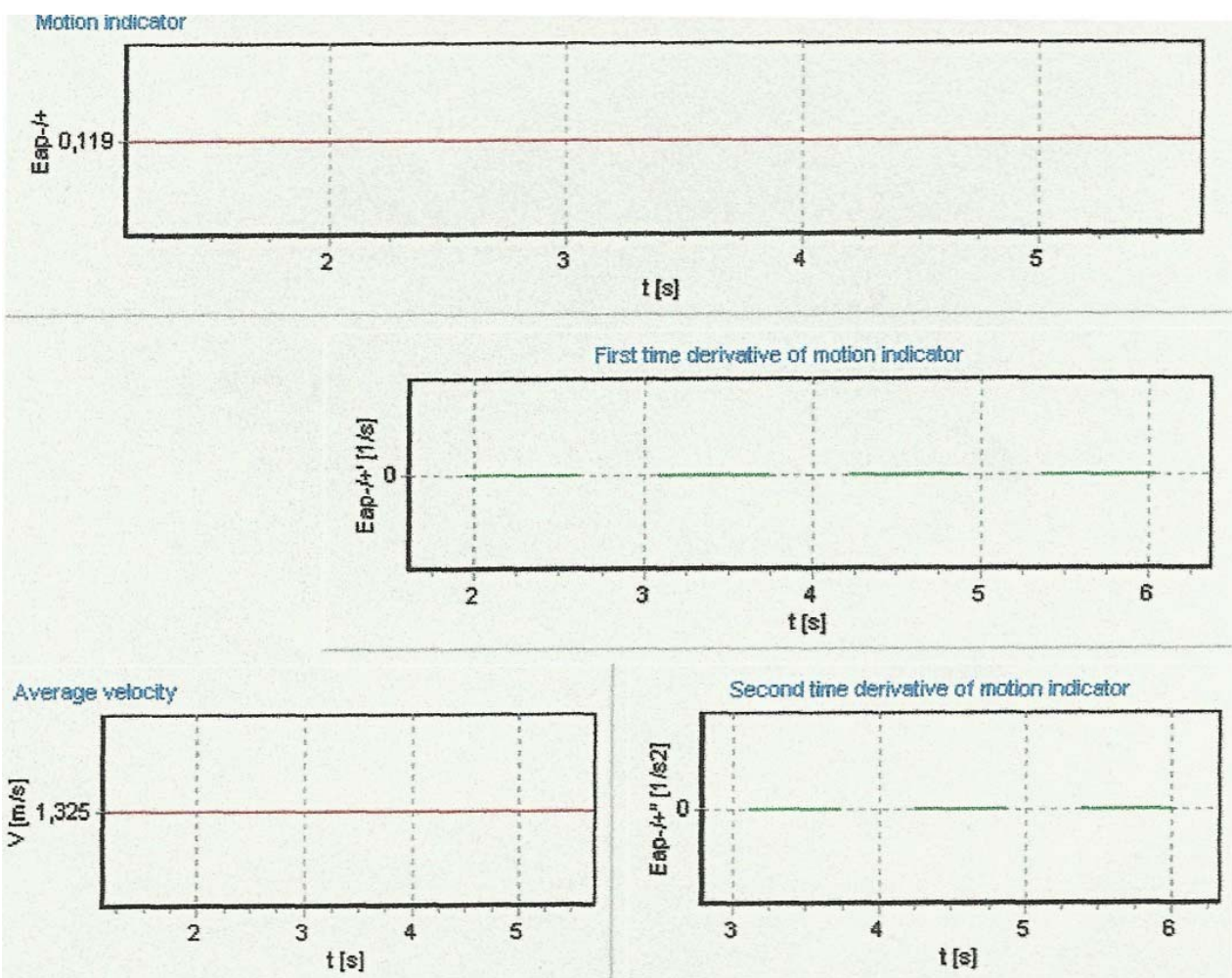

Fig.5. Example 3 - steady locomotion with constant demand for the amount of medium power.
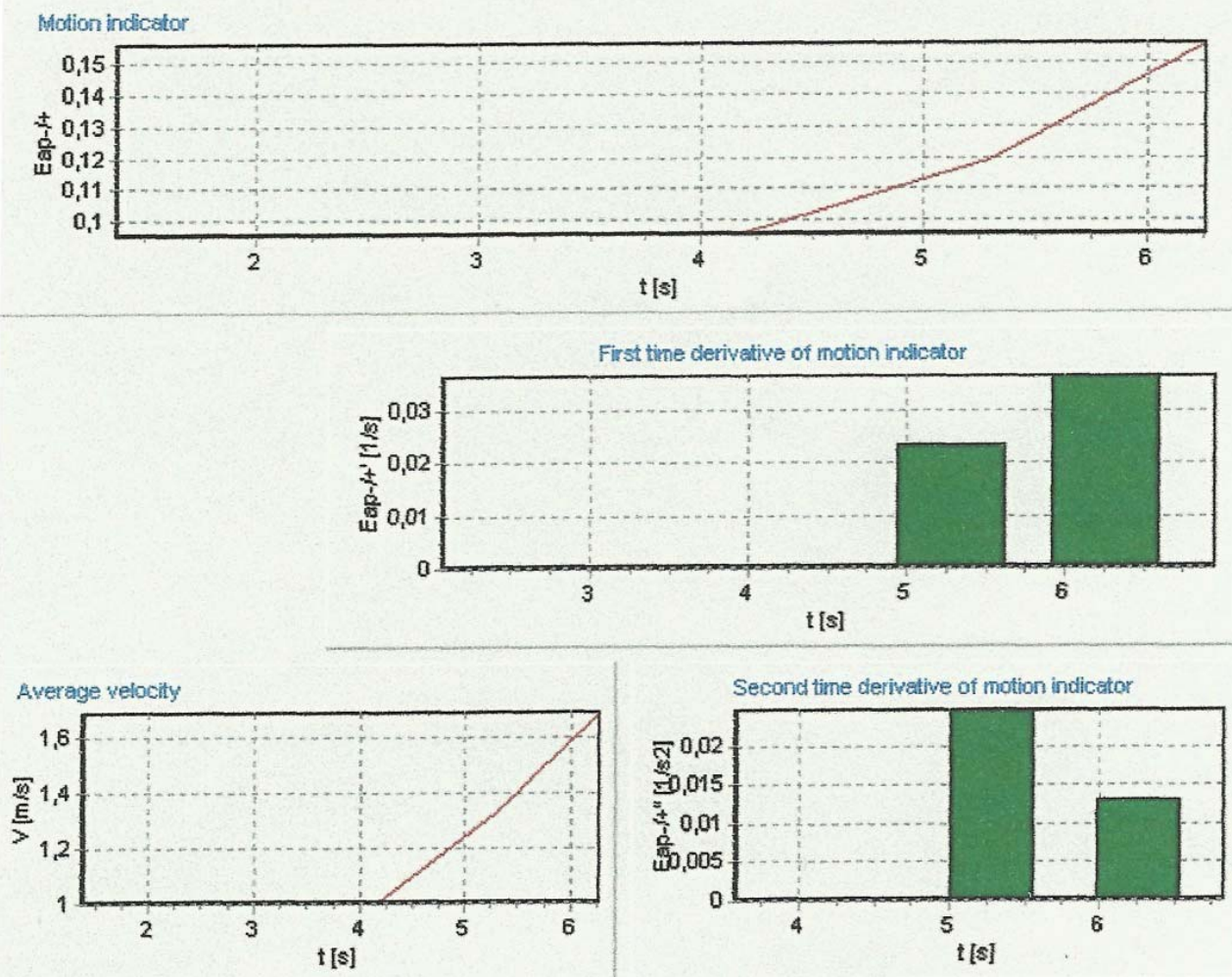

Fig.6. Example 4 - non-steady locomotion, chaotically determined (this configuration is the most similar to steady locomotion with constant - linear increase - demand for the amount of medium power. 


\section{Conclusions}

The method introduced here is another step in human gait analysis. It goes beyond the current canon of human locomotion (single step analysis only) and is slowly approaching an essential issue - gait investigation in natural conditions. The method is not yet completely "on-line", it still needs devices, which would be able to collect data from one human step during multistep gait. We can at last register these steps (separately) for variable locomotion velocity and frequency.

Moreover, a method used to calculate two time derivatives of the motion indicator cannot determine the value of the first time derivative after the first step and the value of the second time derivative after the first two steps. It appears that it is the case because there are no gait parameters in the initial conditions for time $t_{0}$.

Another stage in multistep human locomotion research should be adopting the DChC program to work "on-line" - reading data from a subject during normal gait and solving equations in "real-time".

\section{References}

Jaworek K. (1992): Method of evalouation of human locomotion for running and walking. - Work of Institute of Biocybernetics and Biomedical Engineering Polish Academy of Sciences, vol.32, Warsaw.

Jaworek K. (2006): Two investigatory hypothesis in biomechanics of human locomotion. - Making of VII Scientific Seminar Mechanics in Medicine, October, Boguchwała, pp.93-102.

Jaworek K., Derlatka M. and Dominikowski M. (1998): On an investigation of human locomotion by Penny and Giles electrogoniometer. - Proc. ed. by Christian Hogfors, vol.12, University of Goeteberg, Sweden, pp.21-27.

Jaworek K. and Ferenc A. (1992): A cordless electrogoniometer for simplified gait analysis. - Proc. of International Centre of Biocybernetics IBiB-PAN, Warsaw, pp.53-63.

Winter D.A. (1991): The Biomechanics and Motor Control of Human Gait: Normal, Elderly and Pathological, (second edition). - Univeristy of Waterloo Press.

Żur K.K. (2011): A new idea of modelling and dynamic analysis of human walking apparatus. - Acta Mechanica et Automatica, No.1, Białystok, pp.148-153.

Żur K.K. and Jaworek K.A. (2011): New idea of control of walking anthropomophic robot DAR type, "Measurments.Control.Robotic". - Industrial Research Institute for Automation and Measurements, No.2, Warsaw, pp.462-473.

Received: March 30, 2013

Revised: June 28, 2013 